\title{
EXACT FORMULAE FOR VARIANCES OF FUNCTIONALS OF CONVEX HULLS
}

\author{
CHRISTIAN BUCHTA, ${ }^{*}$ Salzburg University
}

\begin{abstract}
The vertices of the convex hull of a uniform sample from the interior of a convex polygon are known to be concentrated close to the vertices of the polygon. Furthermore, the remaining area of the polygon outside of the convex hull is concentrated close to the vertices of the polygon. In order to see what happens in a corner of the polygon given by two adjacent edges, we consider-in view of affine invariance $-n$ points $P_{1}, \ldots, P_{n}$ distributed independently and uniformly in the interior of the triangle with vertices $(0,1)$, $(0,0)$, and $(1,0)$. The number of vertices of the convex hull, which are close to the origin $(0,0)$, is then given by the number $\widetilde{N}_{n}$ of points among $P_{1}, \ldots, P_{n}$, which are vertices of the convex hull of $(0,1), P_{1}, \ldots, P_{n}$, and $(1,0)$. Correspondingly, $\widetilde{D}_{n}$ is defined as the remaining area of the triangle outside of this convex hull. We derive exact (nonasymptotic) formulae for $\operatorname{var} \widetilde{N}_{n}$ and $\operatorname{var} \widetilde{D}_{n}$. These formulae are in line with asymptotic distribution results in Groeneboom (1988), Nagaev and Khamdamov (1991), and Groeneboom (2012), as well as with recent results in Pardon (2011), (2012).
\end{abstract}

Keywords: Convex hull; random point; variance

2010 Mathematics Subject Classification: Primary 60D05

Secondary 52A22

\section{Introduction}

Let $N_{n}$ denote the number of vertices of the convex hull of a uniform sample of $n$ points from the interior of a convex polygon with $r(\geq 3)$ vertices. A classical result by Rényi and Sulanke [23] states that $\mathbb{E} N_{n}=\frac{2}{3} r \log n+O(1)$ as $n \rightarrow \infty$. For a quarter of a century, in spite of many efforts, cf. e.g. [16, p. 547] and [17, p. 424], essentially no progress has been achieved concerning the variance or the distribution of $N_{n}$. Then Groeneboom [14] succeeded in proving that

$$
\frac{N_{n}-(2 / 3) r \log n}{\sqrt{(10 / 27) r \log n}} \stackrel{\mathrm{D}}{\rightarrow} \mathcal{N}(0,1)
$$

as $n \rightarrow \infty$, where $\stackrel{\text { D }}{\rightarrow}$ ' denotes convergence in distribution and $\mathcal{N}(0,1)$ is the standard normal distribution. Furthermore, he obtained a corresponding result for the unit disk. Groeneboom's statement in the introduction of his paper that 'the results throw light on the rather old problem of the variance structure of the counting process, counting the number of vertices of (parts of) the boundary of the convex hull' is justified.

Rényi and Sulanke, in a second paper [24], also studied the area of the convex hull of a uniform sample of $n$ points from the interior of a square. In [4] their result was extended

Received 15 November 2011; revision received 23 October 2012.

* Postal address: Department of Mathematics, Salzburg University, Hellbrunner Straße 34, 5020 Salzburg, Austria. Email address: christian.buchta@sbg.ac.at 
to a convex polygon with $r(\geq 3)$ vertices. Here we write $A\left(P_{r}\right)$ for the area of the convex polygon and $D_{n}$ for the remaining area of the polygon outside of the convex hull. In order to emphasize the similarity of the formulae for the number of vertices of the convex hull on the one hand and the-suitably expressed-area of the convex hull on the other hand, we define the 'rescaled remaining area' by $\bar{D}_{n}=n\left(A\left(P_{r}\right)\right)^{-1} D_{n}$. In this notation the result says that $\mathbb{E} \bar{D}_{n}=\frac{2}{3} r \log n+O(1)$ as $n \rightarrow \infty$. Nagaev and Khamdamov [19] extended Groeneboom's result for the number of vertices of the convex hull to the joint distribution of the number of vertices and the (rescaled remaining) area of the convex hull. They proved that

$$
\left(\frac{N_{n}-(2 / 3) r \log n}{\sqrt{(10 / 27) r \log n}}, \frac{\bar{D}_{n}-(2 / 3) r \log n}{\sqrt{(28 / 27) r \log n}}\right) \stackrel{\mathrm{D}}{\rightarrow} \mathcal{N}(\mathbf{0}, \Sigma),
$$

where $\mathcal{N}(\mathbf{0}, \Sigma)$ is the two-dimensional normal distribution with expectation the zero vector and covariance matrix $\Sigma$ given by

$$
\Sigma=\left(\begin{array}{cc}
1 & \sqrt{\frac{5}{14}} \\
\sqrt{\frac{5}{14}} & 1
\end{array}\right)
$$

Independently, Cabo and Groeneboom [12] derived the corresponding limit theorem just for the area. (See the discussion in [6]. In 2006, Groeneboom informed me in a private correspondence that the constant under the root in the denominator in the limit theorem stated on page 43 of [12] is the consequence of an incorrect application of the time reversal argument used on page 46 of [12]. The correct application of the argument yields the constant $\frac{28}{27}$, in accordance with the result by Nagaev and Khamdamov.)

As for the similarity of the results for the number of vertices and for the area, Efron [13] proved the identity $V(C)^{-1} \mathbb{E} D_{n}=(n+1)^{-1} \mathbb{E} N_{n+1}$ for a uniform sample of $n$ points from the interior of an arbitrary convex body $C$ with volume $V(C)$. In [6] this identity was extended from expected values to variances and higher moments. The relation between the number of vertices and the area exhibited by the result of Nagaev and Khamdamov [19] was recently reinvestigated by Groeneboom [15]. This paper also comments on a further paper of Nagaev [18].

The limit theorems mentioned were achieved by approximating the process of vertices of the convex hull by the process of extreme points of a Poisson point process. The most intricate part is the computation of the variance of the random variable associated with the approximating process of extreme points. Deriving the variance in an elementary way, we replace tremendous calculations by simple conclusions.

Recently, Pardon [20], [21] proved, for the convex hull of a uniform sample of $n$ points from the interior of an arbitrary plane convex set of unit area, that the distribution of $\left(N_{n}-\right.$ $\left.\mathbb{E} N_{n}\right) / \sqrt{\operatorname{var} N_{n}}$ as well as the distribution of $\left(D_{n}-\mathbb{E} D_{n}\right) / \sqrt{\operatorname{var} D_{n}}$ tend to the standard normal distribution and that

$$
\mathbb{E} N_{n} \asymp \operatorname{var} N_{n} \asymp n \mathbb{E} D_{n} \asymp n^{2} \operatorname{var} D_{n}
$$

as $n \rightarrow \infty$.

Further important results were recently obtained by Schreiber and Yukich [28], Bárány and Reitzner [2], and Bárány and Steiger [3]. New surveys about the convex hull of random points are due to Reitzner [22] and Schneider [26]. Many references are also contained in the monograph by Schneider and Weil [27], as well as in [10] and [11]. 


\section{The variance of the number of vertices}

Groeneboom's limit theorem, for the number of vertices of the convex hull in the case of a convex polygon with $r$ vertices (see Corollary 2.5 of [14]), is a consequence of the limit theorem in the particular case of the unit square (see Corollary 2.4 of [14]), which in turn is a consequence of Theorem 2.4 of [14]. This fundamental theorem of Groeneboom's paper provides formulae for the expected value and for the variance of the number of extreme points of the 'left-lower' boundary of the convex hull of a Poisson point process on $\mathbb{R}_{+}^{2}$, the positive quadrant, with intensity Lebesgue measure. Now, instead of investigating the approximating random variable associated with the Poisson point process, we deal directly with the number of vertices of the 'left-lower' boundary of the convex hull of a uniform sample from the interior of the unit square.

It is well known that the vertices of the convex hull of a uniform sample from the interior of a convex polytope are 'concentrated' close to the vertices of the polytope; cf. in particular [1], where the notion 'concentration of vertices' is also explained in detail. Hence, among the vertices of the 'left-lower' boundary of the convex hull of a sample from the interior of the unit square, only those which are close to the origin $(0,0)$ matter asymptotically. (We think of the unit square with vertices $(0,0),(0,1),(1,0)$, and $(1,1)$.)

The vertices of the convex hull, which are close to the origin, can be captured in the following way: take a line intersecting both the positive $x$-axis and the positive $y$-axis, and consider the convex hull of the two intersection points and those of the points of the sample which lie on the same side of the line as the origin. We are free to take the line through the points $(0,1)$ and $(1,0)$. Furthermore, it does not matter whether a sample is taken straightaway from the interior of the triangle with vertices $(0,1),(0,0)$, and $(1,0)$, instead of considering only the points among a sample from the interior of the square, which fall into the interior of the triangle. (Again, cf. [1].)

Thus we are led to the following task: assume that $n$ points $P_{1}, \ldots, P_{n}$ are distributed independently and uniformly in the interior of the triangle with vertices $(0,1),(0,0)$, and $(1,0)$. Consider the convex hull of $(0,1), P_{1}, \ldots, P_{n}$, and $(1,0)$. Denote by $\widetilde{N}_{n}$ the number of those points among $P_{1}, \ldots, P_{n}$ which are vertices of the convex hull. What is the variance of this number?

The distribution of the random variable $\tilde{N}_{n}$ was determined explicitly in [7]. There the probability $p_{k}^{(n)}(k=1, \ldots, n)$ that $\tilde{N}_{n}=k$ was obtained in the form

$$
p_{k}^{(n)}=\sum \frac{i_{1} \cdots i_{k}}{\left(\begin{array}{c}
i_{1}+1 \\
2
\end{array}\right)\left(\begin{array}{c}
i_{1}+i_{2}+1 \\
2
\end{array}\right) \cdots\left(\begin{array}{c}
i_{1}+\cdots+i_{k}+1 \\
2
\end{array}\right)},
$$

where the sum is taken over all $i_{1}, \ldots, i_{k} \in \mathbb{N}$ such that $i_{1}+\cdots+i_{k}=n$. Theorem 1 states to what extent the first and the second moment of $\widetilde{N}_{n}$ increase if a further random point-chosen independently and according to the same distribution-is added.

Theorem 1. The differences $\Delta \mathbb{E} \tilde{N}_{n}:=\mathbb{E} \widetilde{N}_{n+1}-\mathbb{E} \tilde{N}_{n}$ and $\Delta \mathbb{E} \tilde{N}_{n}^{2}:=\mathbb{E} \tilde{N}_{n+1}^{2}-\mathbb{E} \tilde{N}_{n}^{2}$ are given by

(i) $\Delta \mathbb{E} \tilde{N}_{n}=\frac{2}{3} \frac{1}{n+1}$,

(ii) $\Delta \mathbb{E} \widetilde{N}_{n}^{2}=\frac{8}{9} \frac{1}{n+1} \sum_{k=1}^{n+1} \frac{1}{k}+\frac{10}{27} \frac{1}{n+1}+\frac{4}{9} \frac{1}{n+2}$. 
Immediate consequences are the formulae

$$
\mathbb{E} \widetilde{N}_{n}=\mathbb{E} \widetilde{N}_{1}+\sum_{k=1}^{n-1} \Delta \mathbb{E} \widetilde{N}_{k}=\frac{2}{3} \sum_{k=1}^{n} \frac{1}{k}+\frac{1}{3}
$$

and

$$
\mathbb{E} \tilde{N}_{n}^{2}=\mathbb{E} \tilde{N}_{1}^{2}+\sum_{k=1}^{n-1} \Delta \mathbb{E} \tilde{N}_{k}^{2}=\frac{8}{9} \sum_{k=1}^{n} \frac{1}{k} \sum_{j=1}^{k} \frac{1}{j}+\frac{22}{27} \sum_{k=1}^{n} \frac{1}{k}-\frac{25}{27}+\frac{4}{9} \frac{1}{n+1} .
$$

The resulting surprisingly simple formula

$$
\operatorname{var} \widetilde{N}_{n}=\frac{10}{27} \sum_{k=1}^{n} \frac{1}{k}+\frac{4}{9} \sum_{k=1}^{n} \frac{1}{k^{2}}-\frac{28}{27}+\frac{4}{9} \frac{1}{n+1}
$$

shows that $\operatorname{var} \widetilde{N}_{n} \sim \frac{10}{27} \log n$ as $n \rightarrow \infty$. The formulae constitute an elementary counterpart to the fundamental Theorem 2.4 of [14]. (Also see [12, p. 37].) The same approach can be used to obtain higher moments of $\widetilde{N}_{n}$; however, the technical details are more involved and are therefore elaborated in a separate paper [9].

Proof of Theorem 1. The starting point for our considerations is the formula for the probability $p_{k}^{(n)}$ that $\widetilde{N}_{n}=k, k=1, \ldots, n$. If $n$ is replaced in the formula by $n+1$, the index $i_{k}=n+1-i_{1}-\cdots-i_{k-1}$ attains the integers from 1 to $(n+1)-(k-1)$. Correspondingly, the sum of the remaining indices $j:=i_{1}+\cdots+i_{k-1}$ is at least $k-1$ and at most $n$. Therefore, $p_{k}^{(n+1)}$ can alternatively be written in the form

$$
p_{k}^{(n+1)}=\sum_{j=k-1}^{n} \frac{(n+1-j)}{\left(\begin{array}{c}
n+2 \\
2
\end{array}\right)} \sum \frac{i_{1} \cdots i_{k-1}}{\left(\begin{array}{c}
i_{1}+1 \\
2
\end{array}\right) \cdots\left(\begin{array}{c}
i_{1}+\cdots+i_{k-1}+1 \\
2
\end{array}\right)},
$$

where the second sum is taken over all $i_{1}, \ldots, i_{k-1} \in \mathbb{N}$ such that $i_{1}+\cdots+i_{k-1}=j$. Thus,

$$
p_{k}^{(n+1)}=\frac{1}{\left(\begin{array}{c}
n+2 \\
2
\end{array}\right)} \sum_{j=k-1}^{n}(n+1-j) p_{k-1}^{(j)},
$$

with $p_{0}^{(0)}=1$ and $p_{0}^{(j)}=0$ for $j \in \mathbb{N}$.

The arising recurrence relation is easily translated into a recurrence relation for expected values. Interchanging the order of summation, we obtain

$$
\begin{aligned}
\mathbb{E} \tilde{N}_{n+1} & =\sum_{k=1}^{n+1} k p_{k}^{(n+1)} \\
& =1+\frac{1}{\left(\begin{array}{c}
n+2 \\
2
\end{array}\right)} \sum_{j=1}^{n}(n+1-j) \sum_{k=1}^{j} k p_{k}^{(j)} \\
& =1+\frac{1}{\left(\begin{array}{c}
n+2 \\
2
\end{array}\right)} \sum_{j=1}^{n}(n+1-j) \mathbb{E} \tilde{N}_{j} .
\end{aligned}
$$


Immediate consequences are the identity

$$
\left(\begin{array}{c}
n+2 \\
2
\end{array}\right) \mathbb{E} \widetilde{N}_{n+1}-\left(\begin{array}{c}
n+1 \\
2
\end{array}\right) \mathbb{E} \widetilde{N}_{n}=n+1+\sum_{j=1}^{n} \mathbb{E} \widetilde{N}_{j}
$$

as well as the further identity

$$
\left(\left(\begin{array}{c}
n+2 \\
2
\end{array}\right) \mathbb{E} \tilde{N}_{n+1}-\left(\begin{array}{c}
n+1 \\
2
\end{array}\right) \mathbb{E} \tilde{N}_{n}\right)-\left(\left(\begin{array}{c}
n+1 \\
2
\end{array}\right) \mathbb{E} \tilde{N}_{n}-\left(\begin{array}{l}
n \\
2
\end{array}\right) \mathbb{E} \tilde{N}_{n-1}\right)=1+\mathbb{E} \tilde{N}_{n}
$$

It is essential to note that the last identity can equivalently be written in the form

$$
\left(\begin{array}{c}
n+2 \\
2
\end{array}\right)\left(\mathbb{E} \tilde{N}_{n+1}-\mathbb{E} \tilde{N}_{n}\right)-\left(\begin{array}{l}
n \\
2
\end{array}\right)\left(\mathbb{E} \tilde{N}_{n}-\mathbb{E} \tilde{N}_{n-1}\right)=1,
$$

such that

$$
\Delta \mathbb{E} \tilde{N}_{n}=\frac{(n-1) n}{(n+1)(n+2)} \Delta \mathbb{E} \tilde{N}_{n-1}+\frac{2}{(n+1)(n+2)} .
$$

Consequently,

$$
\Delta \mathbb{E} \widetilde{N}_{n}=\frac{1}{n(n+1)^{2}(n+2)}\left(12 \Delta \mathbb{E} \tilde{N}_{1}+2 \sum_{j=2}^{n} j(j+1)\right) .
$$

Since $\mathbb{E} \tilde{N}_{1}=1, \mathbb{E} \tilde{N}_{2}=\frac{4}{3}$, and $\sum_{j=2}^{n} j(j+1)=\frac{1}{3} n(n+1)(n+2)-2$, we obtain the claimed formula for $\Delta \mathbb{E} \widetilde{N}_{n}$.

The value of $\Delta \mathbb{E} \tilde{N}_{n}^{2}$ can be derived analogously without notable additional difficulties. First we obtain

$$
\begin{aligned}
\mathbb{E} \widetilde{N}_{n+1}^{2} & =\sum_{k=1}^{n+1} k^{2} p_{k}^{(n+1)} \\
& =1+\frac{1}{\left(\begin{array}{c}
n+2 \\
2
\end{array}\right)} \sum_{j=1}^{n}(n+1-j) \sum_{k=1}^{j}\left(k^{2}+2 k\right) p_{k}^{(j)} \\
& =1+\frac{1}{\left(\begin{array}{c}
n+2 \\
2
\end{array}\right)} \sum_{j=1}^{n}(n+1-j)\left(\mathbb{E} \widetilde{N}_{j}^{2}+2 \mathbb{E} \widetilde{N}_{j}\right)
\end{aligned}
$$

Then conclusions corresponding to those above yield

$$
\begin{aligned}
\left(\begin{array}{c}
n+2 \\
2
\end{array}\right)\left(\mathbb{E} \tilde{N}_{n+1}^{2}-\mathbb{E} \tilde{N}_{n}^{2}\right)-\left(\begin{array}{l}
n \\
2
\end{array}\right)\left(\mathbb{E} \tilde{N}_{n}^{2}-\mathbb{E} \tilde{N}_{n-1}^{2}\right) & =1+2 \mathbb{E} \tilde{N}_{n} \\
& =1+2\left(\mathbb{E} \tilde{N}_{1}+\sum_{k=1}^{n-1} \Delta \mathbb{E} \tilde{N}_{k}\right) \\
& =\frac{4}{3} \sum_{k=1}^{n} \frac{1}{k}+\frac{5}{3},
\end{aligned}
$$


such that

$$
\Delta \mathbb{E} \widetilde{N}_{n}^{2}=\frac{(n-1) n}{(n+1)(n+2)} \Delta \mathbb{E} \widetilde{N}_{n-1}^{2}+\frac{2}{(n+1)(n+2)}\left(\frac{4}{3} \sum_{k=1}^{n} \frac{1}{k}+\frac{5}{3}\right)
$$

and, furthermore,

$$
\Delta \mathbb{E} \widetilde{N}_{n}^{2}=\frac{1}{n(n+1)^{2}(n+2)}\left(12 \Delta \mathbb{E} \widetilde{N}_{1}^{2}+2 \sum_{j=2}^{n} j(j+1)\left(\frac{4}{3} \sum_{k=1}^{j} \frac{1}{k}+\frac{5}{3}\right)\right) .
$$

Since $\mathbb{E} \tilde{N}_{1}^{2}=1, \mathbb{E} \tilde{N}_{2}^{2}=2$, and

$$
\begin{aligned}
\sum_{j=2}^{n} j(j+1) \sum_{k=1}^{j} \frac{1}{k} & =\sum_{k=1}^{n} \frac{1}{k} \sum_{j=k}^{n} j(j+1)-2 \\
& =\frac{n(n+1)(n+2)}{3} \sum_{k=1}^{n} \frac{1}{k}-\frac{(n-1) n(2 n+5)}{18}-2
\end{aligned}
$$

the claimed formula for $\Delta \mathbb{E} \widetilde{N}_{n}^{2}$ also follows.

\section{The variance of the area}

Corresponding to the vertices of the convex hull, the area of the convex polygon, which is not covered by the convex hull, is also concentrated close to the vertices of the polygon. Consequently, it is sufficient to consider the difference $\widetilde{D}_{n}$ of the area $A(T)$ of the triangle with vertices $(0,1),(0,0)$, and $(1,0)$, and the area of the convex hull of $(0,1), P_{1}, \ldots, P_{n}$, and $(1,0)$. An identity relating the $k$ th moment of $D_{n}$ to the first moment of $N_{n+1}$, the first two moments of $N_{n+2}, \ldots$, and the first $k$ moments of $N_{n+k}$ was obtained in [6]. Going through the proof of this identity, we see that it can be adapted in order to express the expected value and the variance of $\widetilde{D}_{n}$ by the first moment of $\widetilde{N}_{n+1}$ and the first two moments of $\widetilde{N}_{n+2}$.

Theorem 2. The expected value and the variance of $\widetilde{D}_{n}$ are given by

(i) $\frac{\mathbb{E} \widetilde{D}_{n}}{A(T)}=\frac{\mathbb{E} \widetilde{N}_{n+1}}{n+1}$,

(ii) $\frac{\operatorname{var} \widetilde{D}_{n}}{A(T)^{2}}=\mathbb{E}\left(1-\frac{\widetilde{N}_{n+2}}{n+1}\right)\left(1-\frac{\widetilde{N}_{n+2}}{n+2}\right)-\left(\mathbb{E}\left(1-\frac{\widetilde{N}_{n+1}}{n+1}\right)\right)^{2}$.

In view of the exact formulae for $\mathbb{E} \tilde{N}_{n+1}, \mathbb{E} \tilde{N}_{n+2}$, and $\mathbb{E} \tilde{N}_{n+2}^{2}$ arising from Theorem 1 , we obtain, as immediate consequences of Theorem 2 , the exact formulae

$$
\frac{\mathbb{E} \widetilde{D}_{n}}{A(T)}=\frac{1}{n+1}\left(\frac{2}{3} \sum_{k=1}^{n+1} \frac{1}{k}+\frac{1}{3}\right)
$$


and

$$
\begin{aligned}
\frac{\operatorname{var} \widetilde{D}_{n}}{A(T)^{2}}=\frac{1}{(n+1)(n+2)}( & \frac{28}{27} \sum_{k=1}^{n+1} \frac{1}{k}+\frac{4}{9} \sum_{k=1}^{n+1} \frac{1}{k^{2}}-\frac{55}{27}-\frac{4}{9} \frac{1}{n+1}\left(\sum_{k=1}^{n+1} \frac{1}{k}\right)^{2} \\
& -\frac{4}{9}\left(\frac{1}{n+1}-\frac{2}{n+2}\right) \sum_{k=1}^{n+1} \frac{1}{k}-\frac{1}{9} \frac{1}{n+1} \\
& \left.+\frac{40}{27} \frac{1}{n+2}+\frac{4}{9} \frac{1}{n+3}+\frac{8}{9} \frac{1}{(n+2)^{2}}\right) .
\end{aligned}
$$

This shows that $A(T)^{-2}$ var $\widetilde{D}_{n} \sim \frac{28}{27} n^{-2} \log n$ as $n \rightarrow \infty$.

Proof of Theorem 2. Denote by $\widetilde{A}_{n}$ the area of the convex hull of $(0,1), P_{1}, \ldots, P_{n}$, and $(1,0)$, where $P_{1}, \ldots, P_{n}$ are $n$ points distributed independently and uniformly in the interior of the triangle with vertices $(0,1),(0,0)$, and $(1,0)$. The arguments in the proof of Theorem 1 of [6] yield

$$
\frac{\mathbb{E} \tilde{A}_{n}}{A(T)}=1-\frac{\mathbb{E} \tilde{N}_{n+1}}{n+1}
$$

and

$$
\frac{\mathbb{E} \widetilde{A}_{n}^{2}}{A(T)^{2}}=\mathbb{E}\left(1-\frac{\widetilde{N}_{n+2}}{n+1}\right)\left(1-\frac{\widetilde{N}_{n+2}}{n+2}\right) .
$$

Since $\widetilde{A}_{n}+\widetilde{D}_{n}=A(T)\left(=\frac{1}{2}\right)$, clearly $\mathbb{E} \widetilde{D}_{n}=A(T)-\mathbb{E} \widetilde{A}_{n}$, and the variance of $\widetilde{D}_{n}$ is just the variance of $\widetilde{A}_{n}$.

\section{Concluding remarks}

The formula for var $\widetilde{N}_{n}$ arising from Theorem 1 was first obtained in 2003 and published without proof in the announcement [5]. Over the intervening years the original proof has been simplified considerably. Tools, which were needed in earlier versions of the proof, could be avoided step by step.

From the exact distribution of $\widetilde{N}_{n}$, the exact distribution of $N_{n}$ can be derived, where $N_{n}$ is the number of vertices of the convex hull of a uniform sample from the interior of a convex polygon with $r(\geq 3$ ) vertices. How to proceed in general is sketched in [7], and the arising distributions in the cases that the polygon is a square or a triangle are stated without proof in the announcement [8]. If the distribution-and, hence, the moments—of $N_{n}$ are known, then the moments - and, in particular, the variance — of $D_{n}$, the remaining area of the polygon outside of the convex hull, are also available according to [6]. The details are beyond the scope of the present paper. Here we only state an arising formula for illustration. Assume that the polygon is a triangle of unit area. As above, put $\bar{D}_{n}:=n D_{n}$. Then

$$
\begin{aligned}
\operatorname{var} \bar{D}_{n}=\frac{n^{2}}{(n+1)(n+2)}( & \left(\frac{28}{9}+\frac{8}{n+1}\right) \sum_{k=1}^{n} \frac{1}{k}-\frac{4}{3} \sum_{k=1}^{n} \frac{1}{k^{2}}-4 \\
& \left.-\frac{4}{n+1}\left(\sum_{k=1}^{n} \frac{1}{k}\right)^{2}-\frac{8}{9} \frac{1}{n+1}+\frac{8}{3} \frac{1}{(n+1)^{2}}\right) .
\end{aligned}
$$

Obviously, $\operatorname{var} \bar{D}_{n}=\frac{28}{9} \log n+O(1)$ as $n \rightarrow \infty$ and, thus, $\operatorname{var} \bar{D}_{n}=\frac{28}{27} r \log n+O(1)$ corresponding to $r=3$ vertices of the triangle. 


\section{Acknowledgements}

At an earlier stage of the paper the summation package Sigma developed by Carsten Schneider (cf. e.g. [25]) and the personal support he provided were of great help. Furthermore, I would like to thank Avik Samandarov for translating the preprint [19] into German and Piet Groeneboom for sending me preprints of [15]. The comments of an anonymous referee improved the structure of the paper and the presentation of the results.

\section{References}

[1] BÁrÁNy, I. AND Buchta, C. (1993). Random polytopes in a convex polytope, independence of shape, and concentration of vertices. Math. Ann. 297, 467-497.

[2] BÁrÁny, I. And Reitzner, M. (2010). On the variance of random polytopes. Adv. Math. 225, 1986-2001.

[3] Bárány, I. And Steiger, W. (2013). On the variance of random polygons. Comput. Geom. 46, $173-180$.

[4] Buchta, C. (1984). Stochastische Approximation konvexer Polygone. Z. Wahrscheinlichkeitsth. 67, $283-304$.

[5] Buchta, C. (2003). On the distribution of the number of vertices of a random polygon. Anz. Österreich. Akad. Wiss. Math.-Natur. Kl. 139, 17-19.

[6] Buchta, C. (2005). An identity relating moments of functionals of convex hulls. Discrete Comput. Geom. 33, 125-142.

[7] Buchta, C. (2006). The exact distribution of the number of vertices of a random convex chain. Mathematika 53, 247-254.

[8] Buchta, C. (2009). On the number of vertices of the convex hull of random points in a square and a triangle. Anz. Österreich. Akad. Wiss. Math.-Natur. Kl. 143, 3-10.

[9] Buchta, C. (2012). On the boundary structure of the convex hull of random points. Adv. Geom. 12, 179-190.

[10] Buchta, C. And Reitzner, M. (1997). Equiaffine inner parallel curves of a plane convex body and the convex hulls of randomly chosen points. Prob. Theory Relat. Fields 108, 385-415.

[11] Buchta, C. And Reitzner, M. (2001). The convex hull of random points in a tetrahedron: Solution of Blaschke's problem and more general results. J. Reine Angew. Math. 536, 1-29.

[12] Cabo, A. J. and Groeneboom, P. (1994). Limit theorems for functionals of convex hulls. Prob. Theory Relat. Fields 100, 31-55.

[13] Efron, B. (1965). The convex hull of a random set of points. Biometrika 52, 331-343.

[14] Groeneboom, P. (1988). Limit theorems for convex hulls. Prob. Theory Relat. Fields 79, 327-368.

[15] Groeneboom, P. (2012). Convex hulls of uniform samples from a convex polygon. Adv. Appl. Prob. 44, 330-342.

[16] Jewell, N. P. and Romano, J. P. (1982). Coverage problems and random convex hulls. J. Appl. Prob. 19, $546-561$.

[17] Jewell, N. P. and Romano, J. P. (1985). Evaluating inclusion functionals for random convex hulls. Z. Wahrscheinlichkeitsth. 68, 415-424.

[18] Nagaev, A. V. (1995). Some properties of convex hulls generated by homogeneous Poisson point processes in an unbounded convex domain. Ann. Inst. Statist. Math. 47, 21-29.

[19] Nagaev, A. V. and Khamdamov, I. M. (1991). Limit theorems for functionals of random convex hulls. Preprint, Institute of Mathematics, Academy of Sciences of Uzbekistan, Tashkent (in Russian).

[20] Pardon, J. (2011). Central limit theorems for random polygons in an arbitrary convex set. Ann. Prob. 39, 881-903.

[21] Pardon, J. (2012). Central limit theorems for uniform model random polygons. J. Theoret. Prob. 25, 823-833.

[22] Reitzner, M. (2010). Random polytopes. In New Perspectives in Stochastic Geometry, Oxford University Press, pp. $45-76$.

[23] RÉnYI, A. And Sulanke, R. (1963). Über die konvexe Hülle von $n$ zufällig gewählten Punkten. Z. Wahrscheinlichkeitsth. 2, 75-84.

[24] Rényi, A. and Sulanke, R. (1964). Über die konvexe Hülle von $n$ zufällig gewählten Punkten. II. Z. Wahrscheinlichkeitsth. 3, 138-147.

[25] Schneider, C. (2007). Symbolic summation assists combinatorics. Sém. Lothar. Combin. 56, 1-36.

[26] Schneider, R. (2008). Recent results on random polytopes. Boll. Unione Mat. Ital. (9) 1, 17-39.

[27] Schneider, R. AND WeIL, W. (2008). Stochastic and Integral Geometry. Springer, Berlin.

[28] Schreiber, T. AND YuKich, J. E. (2008). Variance asymptotics and central limit theorems for generalized growth processes with applications to convex hulls and maximal points. Ann. Prob. 36, 363-396. 